\title{
Challenges and Implemented Solutions for the Oral Cleft Prevention Trial in Brazil
}

\author{
Hrishikesh Chakraborty ${ }^{1,3}$, George Wehby ${ }^{2}$, Norman Goco $^{3}$, Janet Moore ${ }^{3}$, Mark Kindem ${ }^{3}$, \\ Camila Vila-Nova ${ }^{4}$, Rui Pereira ${ }^{5}$, Josiane Souza ${ }^{6}$, Alain Viegas ${ }^{7}$, Temis Felix $^{8}$, \\ DaniloMoretti- Ferreira ${ }^{9}$, Antonio Richieri-Costa ${ }^{10}$ and Jeffrey C. Murray ${ }^{2}$
}

\begin{abstract}
${ }^{1}$ Department of Epidemiology and Biostatistics, University of South Carolina, USA. ${ }^{2}$ Department of Pediatrics, Division of Neonatology, University of lowa, lowa City, IA, USA. ${ }^{3}$ Statistics and Epidemiology, RTI International, Research Triangle Park, NC, USA. ${ }^{4}$ Hospital São Antônio - Obras Sociais Irmã Dulce, Salvador, BA, Brazil. ${ }^{5}$ Hospital Materno-Infantil Fernandes Figueira IMIP, Recife, PE, Brazil. ${ }^{6}$ Hospital do Trabalhador, Curitiba, PR, Brazil. ${ }^{7}$ Hospital Paulo Brum - FUNDEF, Lajeado, RS, Brazil. ${ }^{8}$ Hospital de Clínicas de Porto Alegre, RS, Brazil. ${ }^{9}$ Serviço de Aconselhamento Genético - IBB/UNESP, Botucatu, SP, Brazil. ${ }^{10}$ Hospital de Reabilitação de Anomalias Craniofaciais - HRACIUSP, Bauru, SP, Brazil
\end{abstract}

Clinical Trial ID\#: NCT00397917

\section{A B STRACT}

The Oral Cleft Prevention Program (OCPP) is a double-blind, randomized, two-arm multi-site clinical trial designed to assess the effect of periconceptional daily folic acid supplementation $(4.0 \mathrm{mg}$ vs. $0.4 \mathrm{mg})$ on the recurrence of cleft lip, with or without cleft palate, among women with elevated risk. Conducted in seven sites in Brazil, the OCPP randomized 2,510 women in the trial (1,257 to the $4.0 \mathrm{mg}$ group, and 1,253 to the $0.4 \mathrm{mg}$ group). A Data Coordinating Center (DCC), located in the United States, is responsible for the trial's statistical design and analysis, data collection system, data management and storage, activity coordination, data quality control, and site training. DCCs of international trials, such as the OCPP, face unique implementation challenges due to de-centralized organization. The objective of this paper is to describe these challenges and to present the innovative solutions developed to address them.

Key words: International Multisite Trial; Data Coordinating Center; Oral Cleft; Folic Acid

\section{BACKGROUND}

Aa multisite data coordinating center (DCC) is typically involved in all stages of the trial, including planning and design, trial implementation, data collection, trial closeout, final analysis, and publications. Throughout the trial, the DCC is responsible for all aspects of administrative and logistic coordination, including managing communication between all trialsites, coordinating meetings and conference calls, and documenting trial procedures. ${ }^{[1-4]}$ During the

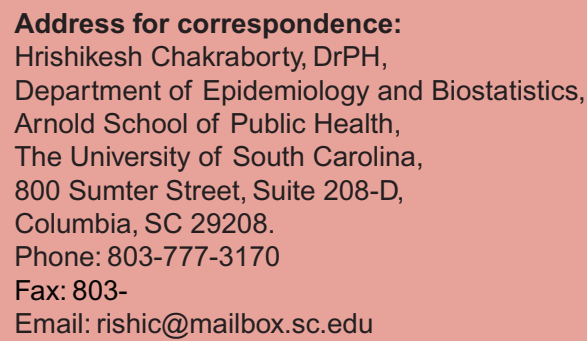

DOI: 10.5530/ijmedph.3.2011.3 planning and design stages of a trial, the DCC is responsible for reviewing the protocol; developingtrial materials; reviewing trial design and sample size calculation; designing and implementing a data management system; coordinating and implementing training of trial staff on study materials and procedures; and ensuring that approvals of all ethical committees are obtained. ${ }^{[1,2]}$ The DCC responsibilities during the data collection stage include reviewing recruitment; randomization; monitoring subject compliance and safety; ensuring data security and quality; producing progress reports; and monitoring of clinical sites. ${ }^{[5-7]}$ At the end of a trial, the DCC oversees final data cleaning, closes the final database, performs statistical analyses, and provides scientific input and coordination for publications.

When investigating less-common diseases, international multisite trials are often required to recruit an adequate sample of participants from each participating site..$^{[1]}$ Such trials face several special challenges not encountered in other trials, such as maintaining efficient communication between sites, facilitating collaboration between the 
investigators, and dealing with cultural differences. ${ }^{[2]}$ The DCC plays a major role in addressing these challenges and facilitating teamwork and cooperation between all of the study site institutions ${ }^{\left[{ }^{[3]}\right.}$ In addition, international multisite trials often face challenges that arise due to differences in regulatory structures, and research infrastructures, which add obstacles to the process of obtaining ethical approval for atrial and may significantly impact a study's timeline. For example, in countries where a national research ethics body exists, approvals for research with human subjects may need to be obtained from these institutions, in addition to local ethical committees at each trial site. ${ }^{[8]}$

The research infrastructures in many developing countries are limited and less developed than those in developed countries, and such infrastructure deficits, if not adequately addressed, can have significant effects on study quality. ${ }^{[0]}$ Thus, the DCCs must be prepared for the workload required to help develop infrastructure and to provide guidance to sites with such needs. ${ }^{[3,10]}$ Language differences also create challenges for DCCs by complicating communications, materials development, and data management. ${ }^{[5]}$

Challenges faced by a DCC when working in developing countries also may arise from a lack of research capacity in those countries. Health research capacity is defined as the ability to define problems, set objectives, build sustainable organizations, and identify solutions to significant national health problems. ${ }^{[1]]}$ Building local health research capacity in developing countries facilitates independent growth, strengthens the international scientific communities, and reduces disparity between developed and developing countries..$^{[2]}$ Health research capacity is also necessary to prevent the global spread of infectious disease and provide countries with the tools to control their health problems. ${ }^{[13]}$ The Global Forum for Health Research stresses that it is important to implement interventions designed to build research capacity in developing countries and to correct the disparity in health research. ${ }^{[14]}$ ADCC's responsibilities on a multisite trial that promotes capacity building, such as the OCPP, often differ from standard multisite studies. The macro-barriers to building health research capacity in developing countries include limited funds in health services; poor infrastructure; political instability; inadequate training and research tools; low priority of research in the health systems; mobile populations; unavailability of up-to-date, population-level health statistics; and scientific isolation and/or movement of local scientists and clinical researchers to developed countries. ${ }^{[1-13,15-17]}$ Working with the study investigators, a DCC can facilitate successful research capacity by establishing a functional infrastructure, obtaining the cooperation of national and local government, providing leadership from experienced health professionals, recruiting and supporting local research workers, developing mentoring partnerships with institutions from developed countries, and providing access to research findings. ${ }^{[1-13,15]}$

The objective of this paper is to describe the Oral Cleft Prevention Program (OCPP) trial design; the challenges faced that have not been described inprevious literature; and the developed innovative solutions to these new challenges. Challenges and implemented solutions are presented for communication, key staff training, IT infrastructure, technical and ethical reviews, materials development, drug manufacturing and labeling, data management and transmission, data quality assurance, and reporting.

\section{ORAL CLEFT PREVENTION PROGRAM}

The OCPPwas a randomized, double-blind study designed to assess the effect of $4 \mathrm{mg}$ and $0.4 \mathrm{mg}$ doses of folic acid, taken on a daily basis during preconception and through the first trimester of pregnancy, by women who themselves have or have had a child with nonsyndromicc left palate $(\mathrm{NSCL} / \mathrm{P})$ and are therefore at higher risk of the recurrence of NSCL/P. ${ }^{[18]}$ NSCL/P status includes cleft cases without other birth defects, significant developmental delay, chromosomal abnormalities, or recognized teratogens. The primary hypothesis is that the higher folic acid dose supplementation $(4.0 \mathrm{mg})$ will decrease the recurrence of NSCL/P in this high-risk group by $50 \%$, compared to the low folic acid dose $(0.4 \mathrm{mg})$. For a $50 \%$ reduction in a baseline rate of $5 \%$ recurrence, 1582 births distributed equally across the two treatment groups are required to have $80 \%$ power with $5 \%$ level of significance.

OCPP study subjects include a convenience sample of women with NSCL/P and/or mothers of at least one child with NSCL/P who currently attend, or have attended in the past, six craniofacial clinics in Brazil for their own care or for the care of their children during the study period and who meet the inclusion criteria. Two models of recruitment_-Outreach and Clinic_-were applied in the trial. (1) The Outreach model was implemented in the city of Bauru (state of São Paulo) through the Hospital de Reabilitação de Anomalias Craniofaciais (HRAC). Study staff primarily recruited subjects through field visits or group meetings with potential subjects at local community facilities. (2) The Clinic-based model was used at HRAC and the following additional clinics: Hospital Santo AntônioObras Sociais Irmã Dulce in Salvador (state of Bahia); Hospital de Clínicas de Porto Alegre in Porto Alegre (state of Rio Grande do Sul); Instituto Materno Infantil Prof. Fernando Figueira-CADEFI/IMIP in Recife (state of Pernambuco); Centro de Atendimento Integrado ao Fissurado Lábio Palatal in Curitiba (state of Paraná); and Fundação para a Reabilitação de Deformidades Crânio- 
Faciais in Lajeado (state of Rio Grande do Sul). Subjects were recruited at the clinic from active patients. Additional recruitment activities included identifying potential subjects from the clinic registry who are not active patients.

Subjects were randomly assigned based on permuted blocks of random sizes and stratified by study clinic to ensure a balanced site representation in both treatment groups. Two possible doses of folic acid, $4.0 \mathrm{mg}$ or $0.4 \mathrm{mg}$, were used; the study pills for both doses were manufactured to be identical in appearance. The study subjects, investigators, and research staff were blinded to the randomization assignments. An 8-week supply of study pills was dispensed to subjects upon enrollment and at regular 8-week intervals to ensure uninterrupted dosing during the preconception and prenatal periods of participation.

Subjects were asked to take a single pill of $4 \mathrm{mg}$ or $0.4 \mathrm{mg}$ of folic acid daily. Pregnant subjects were advised when to stop taking the study pills so that the intervention was limited to the first trimester of pregnancy. Pregnant subjects were monitored by their local prenatal care providers according to the local standard of care. After delivery, the Clinical Coordinator(s) at each site followed the subject and her physician to determine the occurrence of NSCL/P and other birth defects in the infant. The Clinical Coordinator or Co-PrincipalInvestigator at each trial site also evaluated the infant in person for the presence of clefting and other anomalies.

Folate (serum and RBC) levels were assessed at baseline (prior to supplementation) and at least once after that. $\mathrm{B}_{12}$ levels were assessed at baseline and post-supplementation to monitor for $\mathrm{B}_{12}$ deficiency. Subjects were followed up every 2 months post enrollment to deliver the study pills and evaluate their health status, pregnancy occurrence, and compliance with study process. The external Data Safety and Monitoring Board (DSMB) reviewed the adverse events by treatment groups, the observed enrollment rate, and progress on recruitment. The DSMB also reviewed the primary and secondary outcomes by treatment groups at interim looks.

\section{DATA COORDINATION CENTER CHALLENGES AND SOLUTIONS}

RTI International (RTI) served as the DCC for the OCPP, providing training, statistical leadership, data collection and management, and trial communications and logistics, and assisting with materials development. Besides providing central coordination for the OCPP, the OCPP DCC offered data management, documentation, and administrative support to the trial sites. Statistical collaboration included sample size calculations, interim analyses, and reports to the DSMB. The OCPP was developed in collaboration with U.S.-based investigators, Senior Foreign Investigators (SFI), National Institutes of Health (NIH) scientific collaborators, and a team from the DCC (see Figure 1). The RTI DCC team consisted of a senior statistician, trial coordinator, junior statistician, IT expert, and computer programmer.

The DCC encountered several challenges to properly coordinating the trial activities. The early-stage challenges were related to communication, planning, staff training, IT infrastructure, ethical reviews, materials development, drug manufacturing and labeling, the data management system, data quality assurance, and reporting. The following are the specific challenges and adopted solutions.

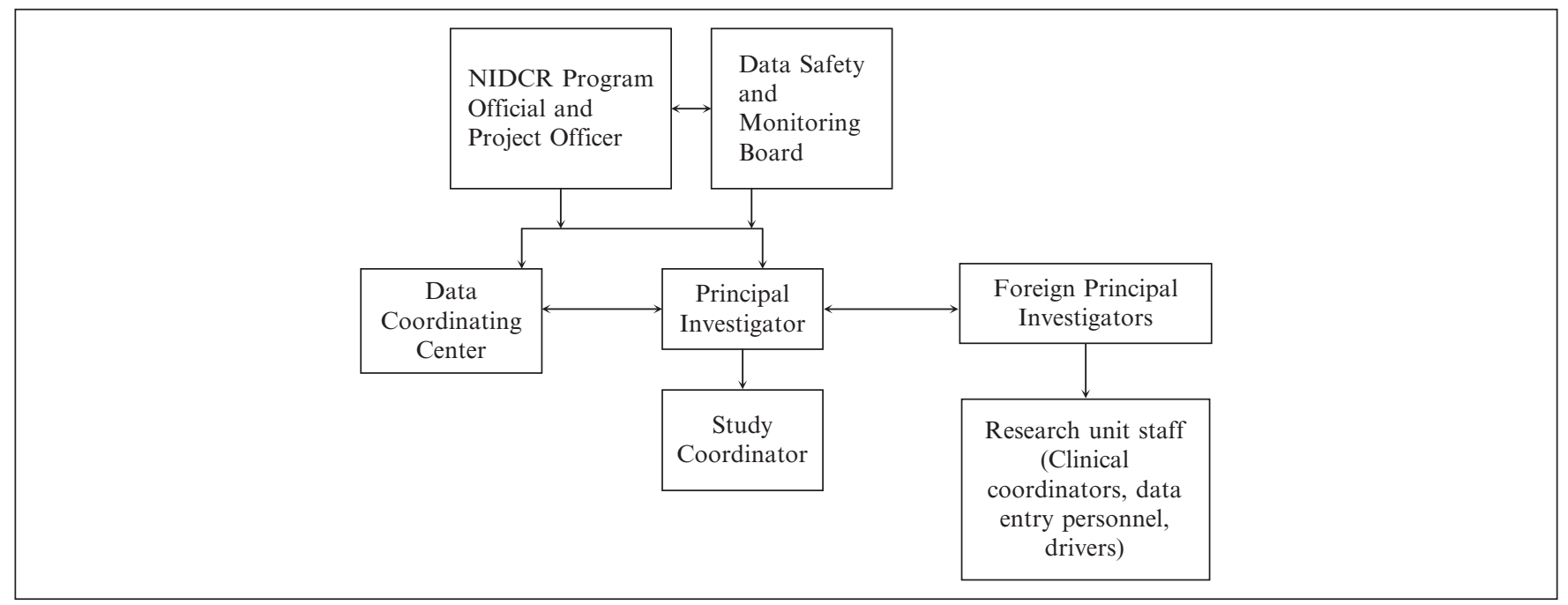

Figure 1: Organizational Chart 


\section{Communication}

At the outset of the OCPP, communication was problematic due to language, distance, and technology. The primary language for communications among study team members and with study participants in Brazil was Portuguese. However, English was the primary language for communicating with the DCC and investigators at the University of Iowa. The lack of Portuguese language capabilities among the DCC and university staff made fluid communications problematic. Teleconferences among the full research team were conducted in English, with translation between English and Portuguese included, particularly when important discussions required investigators to speak in their mother tongue to best express their ideas and when clarification was required. The need for translation during teleconferences tended to increase the duration of discussions. One solution to help improve the teleconferences, particularly for the discussion of study procedures among the study coordinators, was to use small group teleconferences where the research staff in Brazil held discussions only in Portuguese. This provided a comfortable venue for the study coordinators to discuss issues with implementation and facilitate their participation with confidence. In addition to teleconferences, email and instant messaging were used as means of communication among investigators. Instant messaging was of great use for real-time communication for time-dependent queries or concerns with study implementation. Finally, monthly Steering Committee calls were held with the principal investigatos, senior foreign investigators, the NIH program officer, and DCCstaff to discuss study progress. The DCC also had monthly conference calls with the sites to ensure study progression and to clarify adverse events and data issues.

\section{Staff Training}

One of the DCC's first goals with the OCPP was to ensure adequate research training of key staff, especially in the area of clinical trials. To achieve this goal, the DCC, worked with the PIs and the NIHto organize workshops that providedtraining on screening and recruiting trial subjects, randomization, masking, enrollment, data acquisition, data management systems, data quality, and ethics. Additionally, ongoing communicationwas maintained for all staff to discuss reinforcement of protocols, changes in methods, and problems encountered. Lastly, discussionswere held during group conference calls on an as-needed basis.

Strategies for staff training during the implementation of the OCPP included in-person, virtual, and shadowing methods. In-person training was used at the initiation of the trial and again after 4 years. The use of "virtual" or distance training via Web conferencing was used when new clinic sites were brought on or for updates in the study protocol. This method was found to be a cost-effective and flexible solution that limited interruptions in clinic services, which are common when in-person trainings require staff travel. For new sites, virtual training was accompanied by a shadowing method in which staff from the new clinic visited an experienced trial clinic to observe operations. For new sites, in-person training was also conducted, with experienced clinic staff visiting the new clinic to conduct the implementation of the research system.

\section{IT Infrastructure}

The limited IT research infrastructure at certain sites created some challenges. The DCC devised unique solutions to set up an IT infrastructure at each site in order to build a system for day-to-day data management that would endure for several years (see Figure 2). The DCC purchased, configured, shipped, and installed network and server equipment that facilitated collaboration and growth of research activities. To help build the research capacity, in addition to setting up the IT infrastructure, the DCC also hired part-time local staff as network managers who were in charge of providing end-user support, maintaining information systems, and helping with data management activities. These network managers received training from the DCC IT specialists and maintained regular communication through emails, online chat software, and Voice Over Internet Protocol technology.

\section{Technical and Ethical Reviews}

Subsequent to receiving NIH funding through a competitive peer-review process, all foreign funded research undergoes a technical review in Brazil by the Ministry of Health, Science, Technology, and Foreign Affairs (also known as ITAMARATY). The ITAMARATY received the request for OCPP approval through the U.S. State Department at the initiation of the study, and the approval took over a year to complete. Prior to implementation of the OCPP trial-in addition to approvals from the Institutional Review Boards (IRBs) at the University of Iowa and RTI-the ethical review process required two levels of approval in Brazil.The National Commission of Research Ethics (CONEP) in Brazil reviews all study protocols involving foreign collaboration. The CONEP review process is a lengthy process; however, recent regulations for the coordination of multisite trials have facilitated approval. Throughout the life of the project, as amendments to the study protocol were necessary, close and frequent communications with CONEP members helped monitor the status of review and facilitated approvals.

In addition to the CONEP approval, individual research centers in Brazil were required to seek ethics approval from their institutions' Ethics Committees. An institution's Ethics Committee evaluates the appropriateness of its target population and legal information. In the United States, the 


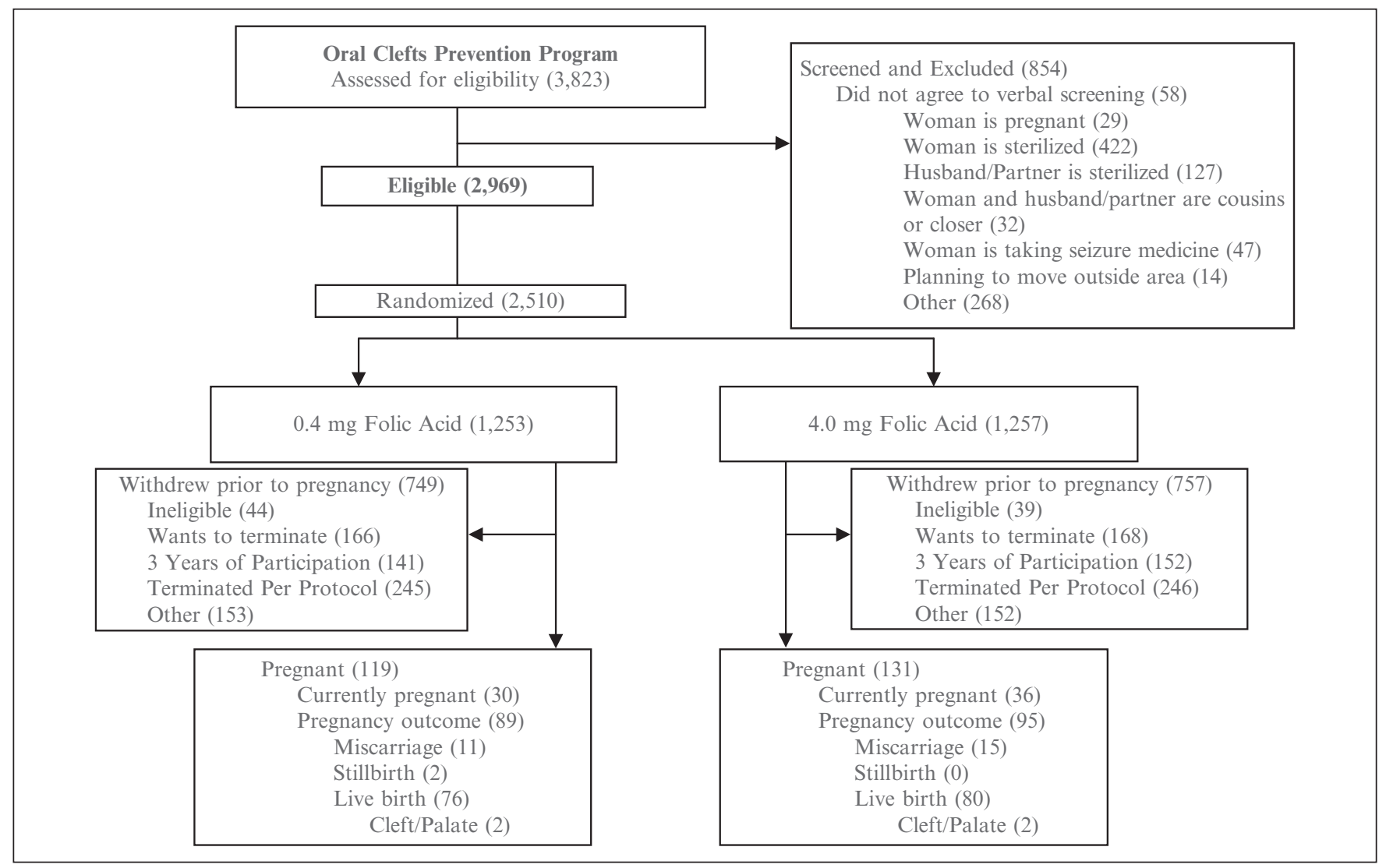

Figure 2: Trial Flow Chart

IRBs of the University of Iowa and RTI, and the DSMB, provided ethics oversight for the trial. In addition, each year all participating institutions conducted an annual review, as required, for continued approval of the research.

Informed consent requirements may differ between U.S. IRBs and local IRBs, and several iterations of consent documents can be needed to satisfy all requirements while ensuring the document is appropriate for different cultures and reading levels. The DCC assisted the investigators in developing a consent form to meet these needs for the OCPP. In order to ensure that all subjects understood the study and the consent forms adequately, the OCPP assured that the Clinical Coordinators read the consent forms to the subjects.

\section{Materials Development}

The pre-testing of any data collection form is standard procedure for many trials. For the OCPP, the local coinvestigators and study staff pre-tested data forms on paper in the research sites to identify early on any potential problems with the form design. Furthermore, the pre-test was used to identify items that were not culturally sensitive and items that were difficult or impossible to collect. Finally, the DCC and investigators obtained assistance from local staff to translate the study protocols, questionnaires, and consent forms into the local language.

\section{Drug Manufacturing and Labeling}

A key issue faced during the OCPP trial was the procurement of the folic pills for use as the trial study drug. The investigators identified a manufacturer in Brazil (ATIVUS Pharmaceutical Industries; http://www.ativus. com.br/) for pill production and obtained production approvals from Brazil's National Agency of Hygienic Control (ANVISA). Using a single manufacturer for all the sites was essential for ensuring a similar intervention across the various sites. Quality control tests were conducted in the United States to evaluate pill quality by an independent laboratory contracted in the United States. In addition, for each batch of pills manufactured, the DCC performed a quality check of the labeling process.

\section{Data Management and Transmissions}

The DCC encountered unique challenges when developing data management systems (DMS) for the OPCC. Before a DMS is released to the field, DCCs establish and testan IT infrastructure and train the data collection and entry staff at each site on the different features and functions of the DMS. The level of training is geared towards the site personnel's degree of computer knowledge. In addition, DCCs can provide hands-on training, where staff entered practice data that have been set up to trigger various responses from the DMS. After the initial training, 


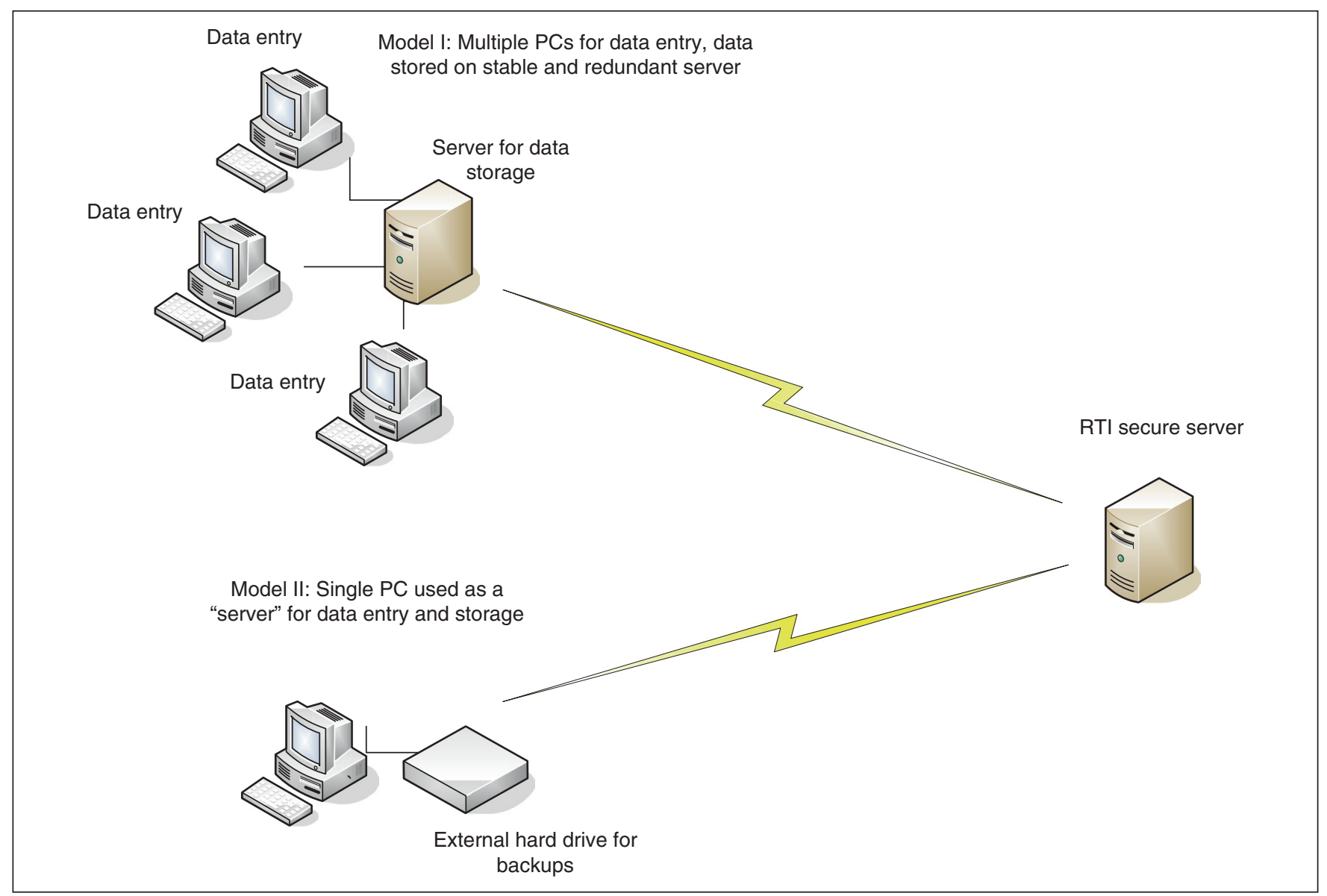

Figure 3: Data Storage and Transmission Flow.

DCCs provide additional technical support remotely through the use of e-mail, instant messaging, and desktop video conferencing. The OPCC DCC (RTI) followed all of these steps for the OPCC study.

Because RTI is based in Research Triangle Park, North Carolina, the use of data transfer protocols over the Internet was the primary method used for the transmission and aggregation of data. However, this process experienced several challenges in operation. For example, Internet connectivity was a problem for some clinics, where access was administered by their respective hospital IT department. At times, authorized ports were closed, causing interruptions in transmission that affected the regular monitoring of data, as well as notification of data-activated alerts (e.g., receipt of adverse events). Poor communication with IT staff at these public facilities also delayed quick resolution. To help better monitor transmissions, the DCC developed weekly transmission reports that tracked the databases received and any updates to key fields. If any sign of delay was identified, the DCC followed up with the clinic site to determine the cause and promptly resolve the issue.

\section{Data Quality Assurance}

To assure data quality, the DCC maintained the central study database, while each study site was responsible for entering and updating all subject records into the local copy of the database. Prior to the start of data collection, the DCC trained site personnel on trial aspects, including requirements for data collection, data recording, data management, and quality control procedures, to ensure uniform understanding of the trial. Once data collection began, the forms were entered on a daily basis in the field. To help ensure accuracy, the data entry program included edit features such as field checks; required data items where entry could not continue until a legitimate value was entered; range checks/valid value checks; within-form consistency checks; logical branching algorithms; across-form checks; and check digit verification on the ID number.

Additionally, on a routine basis, the trial sites ran edit checks that included eligibility checks, data edits, screening log edits, compliance data checks, and laboratory value checks, as well as missing/overdue forms check. Once data were received, the DCC performed additional edit checks; 
statisticians and data management staff worked with the sites to correct the data as needed; and any corrections to the database were accompanied by notes written directly into the database to create an audit trail.

\section{Reporting}

Reporting was an integral part of ensuring the data quality of this study. The DCC collaborated with the trial sites to develop progress reports to ensure that quality data were obtained, analyzed, and disseminated. Monitoring reports were generated routinely on the local level to facilitate monitoring enrollment; study visit tracking (scheduling, missed visits, and contacts); laboratory results; and missing data forms. The ongoing reporting was necessary to allow the DCC to monitor the progress of the study and address any issues related to enrollment, protocol violations, adverse events, and/or data quality as quickly as possible. The DCC generated monthly trial progress report for the monthly SC meeting to evaluate the trial progress. Additionally, the DSMB periodically evaluated the progress of the study, along with the safety and efficacy of the intervention. The DCC collaborated with the Brazil research units to submit DSMB study reports every 6 months. All the serious adverse events were reported to the NIH and the DSMB within 24 hours of receiving the information from the site and were followed until resolution.

\section{DISCUSSION}

As the DCC for OCPP, RTI has faced unique challenges and developed innovative solutions that are resulting inefficient trial coordination and increased research capacity at the sites in Brazil. Many of these challenges arose due to the international differences in regulatory standards and research infrastructure, including deficiencies in IT and clinical trial research training. A main challenge is the lack of technology to coordinate and collect data efficiently. In order to combat the problems, the DCC began by setting up an IT infrastructure at each research site to allow for efficient communication and data collection. Many of the remaining challenges were solved through training sessions with professional staff from the DCC and University of Iowa investigators. A number of the solutions utilized by the OCPP followed similar underlying principles of cultural sensitivity, open communication with research staff, assisting in the long-term capacity building of the sites, and the fostering of relationships. A DCC for an international trial must be flexible and creative to face the unique challenges that will arise and must work with the research sites to develop innovative solutions.

Engaging the local study investigators and staff at Brazil in identifying the challenges and developing solutions was critical for designing effective and sustainable solutions for the challenges at the research site. This step is essential because of the uniqueness and specificity of the priorities, capacities, and challenges at each research site. International research trials require innovative and dynamic data management and study implementation approaches that are able to account not only for "fixed" differences in research environment between countries, but also changing factors that affect study conduct. For example, while seemingly irrelevant for the study, elections of administrators of state and local health departments in Brazil affect the OPCC's ability to reach out through these departments to healthcare providers and patients to inform them about the study. Therefore, designing dynamic approaches that can be systematically evaluated for effectiveness and that change as needed to improve study conduct is instrumental for study success in settings where multiple cultural, social and research-infrastructure factors may have interactive effects on study conduct.

\section{ACKNOWLEDGEMENTS}

This study funded by grants from the National Institute of Child Health and Human Development and the National Institute of Dental and Craniofacial Research (NIDCR), National Institutes of Health (NIH).

\section{REFERENCES}

1. Dzik WH. The NHLBI Clinical Trials Network in transfusion medicine and hemostatis: an overview. J ClinApher. 2006 Apr:21(1):57-9.

2. McDowell I, Helliwell B, Sykes E, Hill G, Lindsay J. Study organization in the Canadian Study of Health and Aging. IntPsychogeriatr. 2001; 13 Supp 1:41-8.

3. Williford WO, Collins JF, Horney A, Kirk G, McSherry F, Spence E, Stinnett S, Howell CL, Garg R, Egan D, Yusuf S; DIG Investigators. The role of the data coordinating center in the DIG trial. Control Clin Trials. 2003 Dec; 24(6 Suppl):277S-288S.

4. Herrell JM, Taylor JA, Gallagher C, Dawud-Noursi S. A multisite study of the effectiveness of methamphetamine treatment: an initiative of the Center for Substance Abuse Treatment. J Psychoactive Drugs. 2000 Apr-Jun; 32(2):143-7.

5. Bangdiwala SI, de Paula CS, Ramiro LS, Munoz SR. Coordination of international multicenter studies: governance and administrative structure. Salud Publica Mex. 2003 Jan-Feb; 45(1):58-66.

6. Kyriakides TC, Babiker A, Singer J, Piaseczny M, Russo J. Study conduct, monitoring and data management in a trinational trial: the OPTIMA model. Clin Trials. 2004; 1(3):277-81.

7. Youngblood ME, Murray KT, Devine E, Latham PK, Hubatch S. Coordinating and monitoring multisite clinical trials that combine pharmacological and behavioral treatments. J Stud Alcohol Suppl. 2005 Jul;(15):82-91; discussion 65.

8. Seal DV, Barry P, Gettinby G, Lees F, Peterson M, Revie CW, Wilhelmus KR; ESCRS Endophthalmitis Study Group. ESCRS study of prophylaxis of postoperative endophthalmitis after cataract surgery: Case for a European multicenter study. J Cataract Refract Surg. 2006 Mar; 32(3):396-406.

9. Abbas EE, Dham P, Shaker D. A multicenter clinical trial in the Arab world. Transplant Proc. 2004 Jul-Aug; 36(6):1801-4.

10. Menezes A, Victora C, Perez-Padilla R, and the PLATINO Team. The Platino project: methodology of a multicenter prevalence survey of chronic 
obstructive pulmonary disease in major Latin American cities. BMC Medical Research Methodology. 2004 June; 4(15).

11. Sitthi-amorn C, Somrongthong R. Strengthening health research capacity in developing countries: a critical element for achieving health equity. BMJ. 2000 Sept: 321

12. Andruchow J, Soskolne C, Racioppi F, and Bertollini R. Capacity building for epidemiologic research: a case study in the newly independent state of Azerbaijan. AEP. 2005 Mar:15(3)

13. Harris E, and Tanner M. Health technology transfer. BMJ. 2000 Sept: 321.

14. Beveridge M, Howard A, Burton K and Holder W. The Ptolemy project: a scalable model for delivering health information in Africa. BMJ. 2003 Oct; 327:790-793
15. Sparks B, and Gupta S. Research in family medicine in developing countries. Ann Fam Med. 2004 May; 2(2):S55-S59.

16. Macklin R. International clinical trials with applicability to mentally impaired individuals: the conundrum of third world sites. Schizophrenia Research. 2001 Mar: 51:87-92

17. Hewson S, Weston J, and Hannah M. Crossing international boundaries: implications for the Term Breech Trial Data Coordinating Centre. Controlled Clinical Trials. 2002 Sept: 23:67-73.

18. Wehby GL, Murray JC. Folic acid and orofacial clefts: a review of the evidence. Oral Dis. 2010 Jan; 16(1):11-9. 